Elżbieta Tomasi-Kapral

\title{
IL RUOLO E LA POSIZIONE DEL TRADUTTORE LETTERARIO NEL XXI SECOLO TRADUTTORE LETTERARIO - IL MEDIATORE TRA LE CULTURE
}

\author{
The role and position of literary translator in the $21^{\text {st }}$ century: \\ a cultural intermediary
}

\begin{abstract}
The $21^{\text {st }}$ century is the age of migration. We observe right now how many people leave their homelands (due to wars and economic situation) and move out to live in places where they cannot communicate using their native language. Therefore the $21^{\text {st }}$ century shall be the age of globalization, formation of multicultural and multilingual societies, giving the newcomers an opportunity to create a new reality. Europe will have to confront with numerous difficulties such as, for instance, creating a space where diversity will not bring about fears or discrimination. On the contrary, it will be accepted and respected. For such a reason there is more and more discussion about cultural exchange. Undoubtedly, one of the windows to look at another culture is literature. The European Commission supports, in recent years, projects focused on literary translation, co-financing the work of translators and their education among the so-called young professionals. Both social and professional position of translators becomes a hot issue of public discussions. It is not an accidental act - together with the changing reality, the role and position of the literature translators in the contemporary world must be redefined. The literary translation brings many benefits and that is the object of this article.

Key words: PETRA, cultural policy, literary translation, TransStar Project, cultural transfer Parole chiave: PETRA, politica culturale, traduzione letteraria, TransStar project, trasferimento culturale

Słowa kluczowe: PETRA, polityka kulturowa, przekład literacki, projekt TransStar, transfer kulturowy
\end{abstract}

\section{Introduzione}

La traduzione letteraria, come anche altre forme di espressione artistica, ha una lunga tradizione. Le prime traduzioni risalgono ai tempi della Roma antica. Molto presto la letteratura e stata riconosciuta come il medium più adatto per conservare 
il pensiero umano, e quindi anche la memoria e la cultura di una nazione ${ }^{1}$. La traduzione di essa veniva (e viene sempre) considerata la chiave per un altro, sconosciuto sistema socioculturale, grazie al quale poteva essere arricchita la cultura della comunità linguistica d'arrivo.

Questa logica la seguì Carlo Magno, per nominare solo un esempio tra i tanti, il quale, nonostante fosse illetterato, all'inizio del suo dominio dette l'impulso a una riforma culturale in più discipline, tra le quali filosofia, letteratura e poesia, la cui riforma è entrata negli annali della storia come «rinascita carolingia». Comprendendo l'importanza della cultura nel processo d'unificazione dell'Impero (il quale in tutta la sua estensione era molto diversificato dal punto di vista culturale), Carlo Magno, con la collaborazione degli intellettuali provenienti da ogni parte dell'impero, cercò quindi di unificare non solo la vita religiosa oppure la giurisprudenza, ma anche la vita culturale, creando un sistema linguistico comprensibile per il popolo non istruito, nel quale venivano tradotte tutte le opere considerate all' epoca importanti.

Presto fu riconosciuto che la traduzione della letteratura era un processo complesso e come tale non consisteva soltanto nel trovare le parole adatte per riportare la stessa cosa, oppure - citando Umberto Eco Dire quasi la stessa $\cos a^{2}$ in un altro sistema linguistico. Gli studiosi delle varie discipline, come ad esempio Cicerone, San Girolamo o Lutero sono giunti alla conclusione che tradurre significava anche, o forse soprattutto, riportare attraverso le parole un intero contesto culturale, sociale, storico, nel quale è stata ancorata la realtà dell'opera letteraria.

Un ragionamento del genere lo ritroviamo anche ad esempio nell'epoca del romanticismo tedesco, dove possiamo osservare una glorificazione dell'immaginazione e della fantasia. Soprattutto i teorici tedeschi svilupparono le teorie secondo le quali il traduttore veniva considerato un genio creativo. Prevaleva anche l'ipotesi che grazie alla sua attività potesse essere arricchita la cultura del sistema di arrivo. Nel 1819 Goethe pubblicò la sua opera intitolata West-Östlicher Divan. Ispirato dal fatto che le grandi opere della letteratura tedesca venivano tradotte nelle lingue orientali, Goethe presentò la sua teoria della natura del processo traduttivo. Postulava quindi la distinzione tra tre tipi di traduzione definiti secondo criteri estetici. Il primo tipo sarebbe la traduzione grazie alla quale il lettore ottiene la possibilità di conoscere attraverso un testo riportato dall'altro sistema linguistico un' altra cultura. Questo sarebbe, secondo Goethe, il merito del traduttore che cerca di esporre le particolarità culturali con i mezzi comprensibili per i suoi lettori, quindi rappresentanti del sistema

1 Cfr. A. Assmann, Erinnerungsräume. Formen und Wandlungen des kulturellen Gedächtnisses, C.H. Beck, München 1999.

${ }^{2}$ U. Eco, Dire quasi la stessa cosa. Esperienze di traduzione, Bompiani, Milano 2012, p. 10. 
culturale d'arrivo. Un altro tipo sarebbe la traduzione definita da Goethe come «l'appropriazione per sostituzione $»^{3}$. Si tratta quindi di un transfer dei fenomeni culturali da un sistema linguistico all'altro, il che secondo Goethe potrebbe però creare una sorte di parodia. Il terzo tipo potrebbe essere secondo il poeta difficile da accettare per i rappresentanti della cultura ricevente. Si tratterebbe infatti di un concetto della traduzione dove il traduttore cercherebbe di mantenere l'identità assoluta tra il testo originale e quello tradotto. Ma grazie a questa fedeltà assoluta, secondo Goethe sarebbe possibile capire il testo originale e il contesto culturale $e^{4}$. Come vediamo, nell'argomentazione di Goethe la questione centrale è dunque non la competenza linguistica del traduttore, ma più che altro la sua capacità di avvicinare il lettore ad un altro sistema culturale. Il ruolo del traduttore letterario non si limita quindi solo a spiegare il significato delle parole, ma a far apparire davanti agli occhi di un lettore tutto ciò che viene spesso nascosto tra le righe.

Alcuni elementi di questo brevemente descritto approccio teorico formulato da Goethe all'inizio del XIX secolo si trovano anche negli altri concetti teorici sviluppati nel XX secolo. Come esempio potrebbe essere citato il concetto di Hönig, secondo il quale "Il traduttore è un costruttore di ponti. Il ponte della traduzione collega due culture e due comunità linguistiche differenti"

Anche Christiane Nord propone un modello di traduzione letteraria basato sul presupposto che la comprensione della realtà fittizia di un' opera letteraria dipenda dal coinvolgimento culturale dei lettori e dalla loro conoscenza del mondo in generale ${ }^{6}$. Visto che sono spesso i particolari eventi storici o fenomeni culturali a dare uno stimolo creativo agli autori, il traduttore deve decidere, come rendere il testo da egli tradotto più comprensibile per i lettori appartenenti alla diversa comunità linguistica, storica e culturale.

Un esempio adeguato in questo contesto potrebbe essere la traduzione del titolo del romanzo Poczatek ${ }^{7}$ scritto da Andrzej Szypiorski nel 1986. Il titolo scelto da Szczypiorski e una metafora che nasconde in sé un ampio contesto storico. La parola «inizio» può significare un nuovo capitolo nella storia della Polonia dopo la seconda guerra mondiale, può significare un nuovo capitolo nelle relazioni tra polacchi ed ebrei dopo la traumatizzante esperienza della Shoah, può significare infine l'inizio di un altro sistema totalitario. Tutte queste possibilità interpretative e associazioni vengono raggruppate in una parola sola,

${ }^{3}$ J. W. Goethe, Berliner Ausgabe. Poetische Werke [v. 1-16], v. 3, Aufbau Verlag, Berlin 1960, p. 308.

${ }^{4}$ Ibidem, p. 307-310.

${ }^{5}$ H. G. Hönig, Konstruktives Übersetzen, Stauffenburg, Tübingen 1995, p. 18.

${ }^{6}$ Ch. Nord, Funktionsgerechtigkeit und Loyalität. Die Übersetzung literarischer und religiöser Texte aus funktionaler Sicht, Frank \& Timme, Berlin 2011, p. 56.

7 A. Szczypiorski, Początek, Instytut Literacki, Paryż 1986. 
non a caso scelta dall'autore come titolo del suo romanzo. Il libro di Szczypiorski fu tradotto nelle diverse lingue, tra le quali tedesco e italiano nel 1988. Sia il traduttore tedesco Klaus Staemmler sia il traduttore italiano Pietro Marchesani hanno rinunciato al titolo scelto dall'autore, sostituendolo con un altro, molto lontano dall'originale: Die schöne Frau Seidenman ${ }^{8}$ nella versione tedesca e La bella signora Seidenman ${ }^{9}$ nella versione italiana.

Il traduttore tedesco giustifica la sua decisione con motivi pragmatici, dicendo: "Con mia moglie abbiamo trovato il titolo Die schöne Frau Seidenman perché pensavamo che quello originale «l'inizio» non attirasse attenzione"10. Prendendo quindi in considerazione le regole del mercato, l'atteggiamento da consumatori dei futuri lettori, ma anche il loro background culturale e contesto sociopolitico, il traduttore ha deciso di cambiare il titolo del libro, convinto che quello originale, tradotto con il suo esatto equivalente linguistico, non avrà in Germania lo stesso effetto come lo aveva nel sistema culturale di partenza. La stessa motivazione vale probabilmente anche per la traduzione in italiano, francese, spagnolo, danese, inglese e altre lingue dell'Europa occidentale. Vale a dire che sia il traduttore ceco sia slovacco hanno tradotto letteralmente il titolo dell'originale, partendo probabilmente dal presupposto che il nuovo lettore, avendo simili esperienze come il lettore polacco, non dovrebbe avere nessuna difficoltà a decodificare la metafora usata da Szczypiorski.

Anche il titolo del libro di Michela Murgia Accabadora ${ }^{11}$ trasmette un ampio contesto culturale e come tale richiede dal traduttore una scelta ben pensata della strategia traduttiva. Con la parola «accabadoras», di provenienza sarda, venivano indicate in Sardegna le donne chiamate per interrompere una lunga agonia che durando più giorni causava al moribondo delle atroci sofferenze. Il ruolo dell'accabadora era quindi quello di effettuare una sorta d'eutanasia. E proprio di queste pratiche parla il romanzo di Michela Murgia. Anche questa volta abbiamo a che fare con un titolo che racchiude in sé un mondo intero, però questa volta non si tratta di un fenomeno comunemente conosciuto, questo contesto culturale è chiaro solo per una piccola parte dei lettori italiani, quelli di provenienza sarda, che conoscono bene diversi aspetti della loro cultura, visto che il fenomeno «accabadora» viene anche oggigiorno trattato in maniera molto discreta e come tale non appare spesso nel discorso pubblico. L'autrice ha scelto quindi quel titolo essendone consapevole, che per la maggior parte dei suoi lettori italiani rimarrà abbastanza enigmatico (il romanzo non è stato

${ }^{8}$ A. Szczypiorski, Die schöne Frau Seidenman, Diogenes Verlag, Zürich 1988.

9 A. Szczypiorski, La bella signora Seidenman, Adelphi edizioni, Milano 1988.

10 A. Fimiak-Chwiłkowska, Das Übersetzen der Kulturbilder. Klaus Staemmlers Übersetzungen der polnischen Literatur, Südwestdeutscher Verlag für Hochschulschriften, Saarbrücken 2013, p. 153.

${ }_{11}$ M. Murgia, Accabadora, Giulio Einaudi editore, Torino 2009. 
scritto nel dialetto sardo, il che permette di presumere che l'autrice si rivolgesse a tutti gli italiani).

Nel 2010 il romanzo di Murgia è stato tradotto in tedesco. La traduttrice Julika Brandestini ha lasciato come titolo della sua traduzione la parola straniera $^{12}$, lanciando in quel modo un chiaro segnale che con quel romanzo il lettore verrà trascinato in uno sconosciuto sistema di credenze, norme sociali e culturali. Nel 2012 il libro di Murgia è stato tradotto anche in polacco. Alla traduttrice Hanna Borkowska i lettori polacchi devono la possibilità di entrare in questo mondo misterioso di credenze popolari sarde. Anche questa volta la traduttrice non rinuncia al titolo originale, però ben sapendo che esso non comunica niente al lettore polacco, aggiunge una spiegazione: Ta, która pomaga odejśćc ${ }^{13}$ provocando così una certa associazione, ma mantenendo nello stesso tempo il background culturale voluto dall'autrice.

Come mostrano i suddetti esempi, le traduzioni dei titoli non dovevano nella maggior parte dei casi essere una copia fedele dell' originale. I traduttori sopraccitati si sono orientati nella scelta della loro strategia traduttiva alla cultura della comunità culturale d'arrivo.

\section{Il traduttore di letteratura esce dall'ombra}

Le suesposte considerazioni mettono in evidenza l' innegabile contributo dei traduttori di letteratura allo scambio interculturale, il che viene considerato molto apprezzabile nel mondo di oggi.

Sta di fatto che il XXI secolo ha portato con se tanti cambiamenti politici e socioculturali, che hanno influenzato anche il mondo delle traduzioni letterarie. Il XXI secolo viene considerato il secolo delle migrazioni. Già oggigiorno stiamo osservando tantissime persone costrette a lasciare i loro paesi (a causa di un conflitto militare oppure della situazione economica) e vivere nelle circostanze, dove non possono comunicare usando la loro lingua madre. Il XXI secolo sarà quindi anche il secolo dell' intensa globalizzazione, di creazione delle società multiculturali e multilingui, nelle quali i rappresentanti delle diverse culture avranno la possibilità di creare insieme una nuova realtà. Come pronosticano sociologi e politici, Europa si confronterà quindi con una serie di problemi tra cui anche quello di creare uno spazio nel quale la diversità non sarebbe temuta o punita, ma riconosciuta e apprezzata. Ė per questo che viene riconosciuta sempre di più l'importanza dello scambio culturale. Senza dubbio la letteratura è una delle piattaforme che ci permette di dare uno sguardo dentro un mondo culturale diverso e sconosciuto.

12 M. Murgia, Accabadora, Klaus Wagenbach Verlag, Berlin 2010.

13 M. Murgia, Accabadora. Ta, która pomaga odejść, Świat Książki, Warszawa 2012. 
Lo scambio culturale è diventato di conseguenza una questione politica. La Commissione europea sostiene quindi progetti nel campo della traduzione letteraria, per esempio finanziando il lavoro dei traduttori sulla traduzione di opere letterarie, o la formazione dei cosiddetti young professionals di quest'arte. Questa non è un'azione casuale - la traduzione letteraria, come è stato illustrato, porta con sé diversi vantaggi, il che è stato riconosciuto al livello di politica europea (ma anche quella mondiale) come mostrano le seguenti citazioni:

Il ricco patrimonio culturale dell'Europa dovrebbe essere accessibile a tutti, non a un'esigua minoranza. Per questo i traduttori sono essenziali ${ }^{14}$.

Condividiamo un solo e medesimo obiettivo: promuovere la disciplina e la pratica della traduzione creativa e assicurare che ottenga maggiore visibilità e apprezzamento in Europa ${ }^{15}$.

Queste opinioni fanno capire che c'è una volontà di effettuare un cambiamento importante. Infatti, l'inizio del XXI secolo ha portato con sé un grande cambiamento di ruolo e status sociale del traduttore letterario. Bisogna infatti constatare, che nonostante la consapevolezza dell'importanza della traduzione letteraria, fino agli anni ottanta e ancora negli anni novanta del secolo scorso il traduttore di letteratura era praticamente inesistente nel dibattito pubblico. La sua situazione di allora rispecchia molto bene il fatto che il suo nome in molti casi non appariva nei libri da egli tradotti. Il traduttore rimaneva invisibile lavorando da solo, nascosto dietro la sua scrivania oppure tra gli scaffali della biblioteca, dove faceva la sua ricerca, esplorando territori sconosciuti, cercando di capire e spesso di tradurre l'intraducibile. Il traduttore di letteratura era anche nella maggior parte dei casi autodidatta. Nei programmi di studi praticamente non c'erano previsti corsi di formazione per i futuri traduttori di letteratura.

Oggigiorno il ruolo del traduttore letterario viene discusso e ridefinito nell'attuale contesto culturale e politico. Un traduttore letterario non è più una persona invisibile, muta, nascosta dietro l'autore. Un traduttore letterario di oggi esce dall'ombra e prende l'iniziativa. Il suo compito non è solo tradurre. Egli prende spesso il ruolo di un manager, che nel fitto delle proposte letterarie deve scoprire quella che potrebbe essere interessante (prendendo in considerazione diversi punti di vista) per il nuovo pubblico. Una volta trovato il testo adatto, il traduttore-manager si mette alla ricerca di una casa editrice, dove la sua traduzione potrebbe essere pubblicata. Entra in trattative, dibatte le condizioni

${ }^{14}$ A. Vassiliou, Le raccomandazioni PETRA Verso nuove condizioni a favore della traduzione letteraria in Europa, Bruxelles 2012, p. 2.

$15 \mathrm{~J}$. M. Barroso, Le raccomandazioni PETRA Verso nuove condizioni a favore della traduzione letteraria in Europa, Bruxelles 2012, p. 8. 
del contratto, della promozione del libro (e quindi anche dello scrittore) per trovare i futuri lettori. Visto così il lavoro del traduttore non consiste solamente nella traduzione, ma richiede numerose capacità al di là delle conoscenze linguistiche, il che viene spesso ignorato nel discorso pubblico.

\section{La traduzione letteraria diventa una questione politica}

La necessità del cambiamento della posizione sociale del traduttore letterario è stata riconosciuta dall'Unione europea per la prima volta nel dicembre 2011. A Bruxelles aveva luogo il primo convegno internazionale PETRA ${ }^{16}$. Tra i partecipanti c'erano 70 organizzazioni provenienti dai paesi europei (non necessariamente stati membri dell'Unione europea) attive nel settore della traduzione letteraria (come per esempio italiano Sindacato Traduttori Editoriali STRADE o polacco Polski Instytut Książki). Durante il convegno sono stati discussi tanti importanti aspetti della traduzione letteraria, tra i quali:

- la formazione del traduttore letterario;

- la gestione del diritto d'autore e dei diritti digitali;

- le politiche editoriali e la situazione sul mercato editoriale;

- lo statuto economico e sociale del traduttore letterario.

Questa iniziativa ci fa capire che è stata riconosciuta la necessità d'introdurre seri cambiamenti riguardanti tanti suesposti aspetti del lavoro, formazione e la situazione giuridica del traduttore letterario.

Nel documento intitolato The PETRA Recommendations - Raccomandazioni PETRA ${ }^{17}$ sono stati quindi raccolti e offerti alle istituzioni europee, nazionali ma anche quelli regionali i suggerimenti su come migliorare la situazione della traduzione letteraria (e anche la sua qualità) e dei traduttori letterari in Europa. Le sopra nominate raccomandazioni sono state presentate nel 2012 alla sopraccitata Commissario europeo per l'istruzione, la cultura, il multilinguismo e la gioventù.

Si parte quindi da due presupposti: come primo vale la pena sottolineare che con questo documento è stato per la prima volta ufficialmente riconosciuto in modo chiaro e inequivocabile il ruolo del traduttore letterario come mediatore interculturale. La letteratura trasmette senza alcun dubbio il patrimonio spirituale dell'umanità, rispecchiando gli importanti processi storici e cambiamenti socioculturali, conserva per le prossime generazioni cosiddetto spirito del tempo, dando così il suo contributo alla creazione della memoria collettiva della nazione. Il che risulta molto importante nel contesto europeo,

16 Piattaforma europea per la traduzione letteraria.

17 Le raccomandazioni PETRA Verso nuove condizioni a favore della traduzione letteraria in Europa, Bruxelles 2012. 
dove patrimonio culturale e la memoria collettiva di una nazione devono essere portati oltre le frontiere (oggi formalmente inesistenti, ma mentalmente sempre presenti).

Come secondo punto viene messo in evidenza un infausto destino del traduttore letterario. Il traduttore è obbligato a porsi al servizio dell'autore del testo originale, quindi è costretto a eclissarsi. Il suo lavoro viene considerato tanto più riuscito quanto più si sarà reso invisibile. Se invece avrà lasciato le tracce visibili nel testo tradotto, diventerà oggetto di critica. In questo senso il traduttore letterario è praticamente relegato nel dimenticatoio ${ }^{18}$.

Il convegno ha dato un importante impulso ai cambiamenti nel ambito delle traduzioni letterarie. Come verrà evidenziato in seguito, è stata riconosciuta la necessità di rivoluzionare certi aspetti della realtà professionale del traduttore. È stato deciso, che per migliorare la situazione del traduttore bisogna garantire al traduttore letterario la stessa visibilità riservata ad ogni creatore. Dovrebbe quindi essere ricordato o citato ovunque sia menzionato l'autore dell'opera da egli tradotta, il che purtroppo oggi non è sempre il caso. Oggi il nome del traduttore appare nella maggior parte dei casi non sulla copertina, ma sulla prima pagina. È sempre un miglioramento, paragonando questa situazione con quella degli anni sessanta o settanta, dove spesso il nome del traduttore non veniva pubblicato oppure veniva pubblicato nei posti poco esposti e quindi invisibili. In questo contesto sembra opportuno citare il punto di vista presentato da Jarniewicz, il quale postula nel suo libro dedicato alla traduzione letteraria ${ }^{19}$ che il nome del traduttore appaia direttamente sulla copertina del libro. Proprio cosi come succede nel caso di audiolibri, dove direttamente sotto il nome dell'autore e il titolo della pubblicazione viene collocata l'informazione su chi ha letto il testo.

Un altro cambiamento postulato che dovrebbe essere effettuato è collegato alla nuova realtà sul mercato editoriale, dove la così detta pubblicazione on-line delle opere letterarie sotto forma di audiolibri oppure nelle biblioteche on-line diventa unalternativa sempre più diffusa (perché più economica) del libro stampato. Nel caso quindi della digitalizzazione di un'opera letteraria dovrebbe essere rispettata la legge sul diritto d'autore anche per il traduttore letterario ${ }^{20}$. Un altro problema messo in discussione risulta dal fatto che la maggior parte della società mondiale non conosce le particolarità collegate al mestiere di traduttore letterario. Bisognerebbe quindi sensibilizzare la critica letteraria alla specificità del lavoro del traduttore letterario evitando cosi le situazioni dove il traduttore viene punito per gli errori commessi dallo scrittore nella sua opera ${ }^{21}$.

${ }^{18}$ Ibidem, p. 37.

19 J. Jarniewicz, Gościnność stowa. Szkice o przekładzie literackim, Wydawnictwo Znak, Kraków 2012, p. 17.

${ }^{20}$ Le raccomandazioni PETRA..., p. 31-32.

${ }^{21}$ Ibidem, p. 38. 
Molto importante, considerando le insufficienti condizioni economiche, è anche la creazione di borse e sussidi finanziari a beneficio del traduttore letterario ${ }^{22}$.

Al di là di quello è molto importante moltiplicare al livello europeo le cosiddette buone pratiche, nello specifico appoggiando le azioni delle associazioni di traduttori letterari, che hanno avuto già successo in altri paesi (come per esempio in Germania l'introduzione di codici di buone pratiche condivise con gli editori). Ci sono anche i tentativi d'introdurre a livello europeo un contratto-tipo le cui clausole rispettino tanto il lavoro del traduttore quanto quello dell'editore ${ }^{23}$.

\section{Concretizzazione dei suggerimenti di PETRA}

Nella discussione sul livello europeo riguardante la posizione di traduttore letterario si nota e si sottolinea anche la necessità di sostenere iniziative nell'intento di realizzare un contesto ideale di lavoro, come per esempio collegi o centri per traduttori ${ }^{24}$. Gli istituti di questo tipo sono molto importanti perché offrono ai professionisti l'opportunità d'immergersi nella cultura straniera, di dialogare con i colleghi, d'incontrarsi e discutere con gli autori oppure di realizzare le ricerche necessarie. In quest'area ci sono già alcuni buoni esempi che possono essere presentati in questo contesto, come La Casa dei Traduttori Looren in Svizzera. Il fatto che la Casa Looren sia situata in Svizzera è dovuto indubbiamente alle particolarità di questo paese. In Svizzera ci sono, come noto, quattro lingue ufficiali, il che influenza in modo marcato il senso di appartenenza nazionale dei membri di questa società. La Casa dei Traduttori Looren, situata in un ambiente tranquillo e idilliaco, offre ai traduttori di letteratura da tutto il mondo tante iniziative diverse nella loro natura, come per esempio programmi, borse, workshops ecc., tra cui non mancano le iniziative dedicate alla lingua italiana, come per esempio Laboratorio italiano. Con laboratorio italiano si intende gli appuntamenti tematici multilingui dedicate sempre ad un tema particolare, attuale nel contesto dello sviluppo della letteratura. I partecipanti - traduttori di madrelingua italiana analizzano e discutono alcune pagine tratte da una delle loro traduzioni in corso d'opera. Lo scopo d'incontri del genere è sempre favorire lo scambio culturale e professionale. Molto importante sono anche eventi pubblici, lavori aperti al pubblico che permettono alla gente comune di partecipare in diretta alla discussione di uno dei testi in lavorazione.

22 Ibidem, p. 50.

23 Ibidem, p. 52.

24 Ibidem, p. $27 \mathrm{sgg}$. 
Anche in Polonia è stato fondato un centro simile - la Villa Decius a Cracovia, la quale offre agli scrittori e traduttori tante possibilità di lavoro, ricerca e sviluppo professionale. Seguendo l'esempio svizzero anche lì i traduttori possono trovare un ambiente adatto alle loro esigenze. La Villa Decius segue i suggerimenti di PETRA offrendo ai traduttori diverse borse di studio, organizzando i simposi tematici, workshop e laboratori. Ideando i loro progetti, gli organizzatori si servono anche dello spazio pubblico di Cracovia. Tante iniziative tra cui incontri con autori e traduttori, presentazioni dei libri tradotti, serate con poetry slam si svolgono nei pub, ristoranti, cinema ecc. situati nel centro storico della città, il che permette di aggiungere un ampio pubblico e di sensibilizzarlo alla specificità del lavoro del traduttore letterario.

Un altro esempio che viene ricordato in questo contesto è La Casa delle Traduzioni situata a Roma. La Casa delle Traduzioni di Roma è però sopratutto un centro di documentazione e consultazione specializzato alla traduzione di ogni tipo. La Casa delle Traduzioni è un progetto nato nel giugno del 2011 con l'obiettivo di valorizzare il ruolo del traduttore e di promuovere la diffusione della lingua e della letteratura italiana nel mondo. Questo centro offre quindi i suoi spazi a traduttori, ricercatori, professionisti dell'editoria e a chiunque sia interessato al mondo della traduzione. C'è però un altro aspetto molto importante: La Casa delle Traduzioni organizza un ampio programma di formazione permanente e di attività culturali rivolte agli specialisti e al pubblico esterno, come per esempio laboratori di traduzione, presentazioni di novità editoriali, giornate di orientamento, incontri e seminari che danno la possibilità ai traduttori italiani e stranieri di confrontarsi e di lavorare insieme. In La Casa delle Traduzioni vengono accolti traduttori da tutto il mondo, il che gli dà l'opportunità di lavorare a contatto diretto con la cultura italiana.

Come si evince da quanto precede, tutte queste iniziative sono destinate a sensibilizzare il pubblico sull'importanza del processo della traduzione letteraria e realizzano le raccomandazioni della soprannominata PETRA. Al primo piano appare pero sempre l'aspetto (inter)culturale e il tentativo di creare una piattaforma di scambio tra le culture, riconoscendolo come il compito fondamentale.

Un altro aspetto essenziale il quale potrebbe migliorare la posizione del traduttore sul mercato del lavoro è la sua formazione. Si pone però la domanda, se le competenze richieste dalla professione così particolare come lo è la professione di traduttore letterario possono essere acquisite attraverso studi universitari oppure per via corsi di formazione. È vero che in questo caso, oltre all'istruzione universitaria (il che vuol dire lo studio pratico e teorico della lingua, letteratura, cultura ecc.) ci vuole anche o sopratutto talento e una certa predisposizione, forma mentis, vocazione che ci spinge verso quell'attività. Quindi gli studi universitari di per sé non bastano, ma di sicuro aiutano nella formazione di young professionals. 
In questo contesto risulta opportuno nominare un'altra iniziativa importante che può essere considerata come un esempio di buone pratiche nell'ambito universitario. Si tratta di un progetto ideato per aiutare i traduttori principianti a capire, come sarà il loro futuro mestiere. Era un progetto finanziato dai fondi europei, previsto per i giovani traduttori di letteratura, che si trovano ancora all'inizio della loro formazione. Il progetto "TransStar Europa" 25 è stato realizzato sia all’Università di Łódź, che all’Università di Tübingen, Praga, Ljubliana, Kiew e Zagreb.

Lo scopo generale di quel vasto progetto consisteva nel preparare i futuri traduttori letterari per il loro ruolo nella società europea, promuovere lo scambio culturale tra i paesi europei, ma anche elaborare dei moduli pratici per la traduzione letteraria ed includerli nei curricula delle università - membri del consorzio. Durante quel triennale progetto si svolgevano regolarmente dei workshop, dove i partecipanti avevano la possibilità di lavorare con i professionisti e stimati traduttori letterari sulla traduzione di testi scritti dagli autori europei contemporanei.

I partecipanti del progetto "TransStar Europa” avevano anche la possibilità di conoscere gli autori delle opere da loro tradotte e preparare insieme a loro delle performance presentate in seguito al pubblico nei diversi paesi europei. Era quindi un' esperienza unica, che permetteva ai giovani traduttori di confrontarsi con i loro primi lettori e di convincersi che l'effetto voluto dallo scrittore è stato ottenuto anche attraverso la loro traduzione. Il progetto era realizzato in un periodo particolare per Europa - nel 2014 la società europea si è confrontata con una realtà che nessuno si aspettava - si tratta dell'invasione russa in Ucraina, uno dei partner nel progetto. Dopo i primi mesi di guerra in Ucraina sono apparse le prime reazioni letterarie da parte degli scrittori e poeti ucraini, i quali, essendo direttamente coinvolti, si sentivano in dovere di esprimersi al riguardo e opporre resistenza alla situazione nel loro paese. Alcuni di questi testi sono stati spontaneamente tradotti dai partecipanti in tutte le lingue rappresentate nel progetto. In tutti i paesi coinvolti nel progetto sono state organizzate le serate intitolate Leggere per L'Ucraina, dove i giovani traduttori hanno presentato i testi letterari da loro tradotti, diventando cosi portavoci degli scrittori, che in quel particolare momento non potevano lasciare il loro paese. Era chiaro per loro che leggendo i testi letterari non avrebbero potuto fermare i panzer russi, ma attraverso queste performance avrebbero potuto far conoscere al pubblico nei 6 paesi europei un problema attuale, prendendo in considerazione diversi punti di vista.

Da quanto precede risulta che i partecipanti del progetto "TransStar Europa" avevano quindi l'opportunità non solo di approfondire la traduzione narrativa

${ }^{25}$ Le informazioni del progetto si trovano sul sito www.transstar-europa.com 
in lezioni teoriche e in laboratori pratici con professionisti affermati nel mondo della traduzione, ma anche di vivere sulla propria pelle la missione del traduttore nella società di oggi.

\section{Conclusioni}

Il prodotto finale della traduzione letteraria è un' opera letteraria e come tale è ancorata in un certo contesto socioculturale. La traduzione letteraria, come risulta dalla sua lunga tradizione, doveva soddisfare diverse esigenze imposte dalle diverse epoche e società. Il mondo di oggi con le sue trasformazioni e i suoi cambiamenti presenta al traduttore le nuove sfide. Il ruolo del traduttore come mediatore tra le culture viene oggigiorno riconosciuto e sostenuto grazie a tante iniziative, come quelle precedentemente menzionate. In alcune teorie moderne della traduzione, come per esempio quella sviluppata da Christine Nord, per la quale il testo letterario non è soltanto un testo poetico, ma anche un testo informativo ${ }^{26}$, viene sottolineata la dimensione pragmatica della letteratura. Il testo letterario deve quindi 'funzionare' non solo nel contesto socioculturale dal quale proviene, ma anche nella cultura ricevente. Se sarà quindi comprensibile per i nuovi lettori dipende non solo dal suo contenuto, ma anche dalle scelte fatte dal traduttore.

\section{Bibliografia e sitografia}

Assmann A., Erinnerungsräume. Formen und Wandlungen des kulturellen Gedächtnisses, C.H. Beck, München 1999.

Barroso J. M., Le raccomandazioni PETRA Verso nuove condizioni a favore della traduzione letteraria in Europa, Bruxelles 2012.

Eco U., Dire quasi la stessa cosa. Esperienze di traduzione, Bompiani, Milano 2012.

Fimiak-Chwilkowska A., Das Übersetzen der Kulturbilder. Klaus Staemmlers Übersetzungen der polnischen Literatur, Südwestdeutscher Verlag für Hochschulschriften, Saarbrücken 2013.

Goethe J. W., Berliner Ausgabe. Poetische Werke [v. 1-16], v. 3, Aufbau Verlag, Berlin 1960.

Hönig H. G., Konstruktives Übersetzen, Stauffenburg, Tübingen 1995.

Jarniewicz J., Gościnność stowa. Szkice o przekładzie literackim, Wydawnictwo Znak, Kraków 2012.

Murgia M., Accabadora, Giulio Einaudi editore, Torino 2009.

Murgia M., Accabadora, Klaus Wagenbach Verlag, Berlin 2010.

Murgia M., Accabadora. Ta, która pomaga odejść, Świat Książki, Warszawa 2012.

${ }^{26}$ Cfr. Ch. Nord, Funktionsgerechtigkeit und Loyalität, Frank \& Timme, Berlin 2011, p. 7. 
Nord Ch., Funktionsgerechtigkeit und Loyalität. Die Übersetzung literarischer und religiöser Texte aus funktionaler Sicht, Frank \& Timme GmbH, Berlin 2011.

Szczypiorski A., Die schöne Frau Seidenman, Diogenes Verlag, Zürich 1988.

Szczypiorski A., La bella signora Seidenman, Adelphi edizioni, Milano 1988.

Szczypiorski A., Poczatek, Instytut Literacki, Paryż 1986.

Vassiliou A., Le raccomandazioni PETRA Verso nuove condizioni a favore della traduzione letteraria in Europa, Bruxelles 2012.

www.transstar-europa.com 